\title{
Interference Management for Co-Channel Mobile Femtocells Technology in LTE Networks
}

\author{
Rand Raheem, Aboubaker Lasebae, Mahdi Aiash, Jonathan Loo \\ School of Science \& Technology, Middlesex University, London, United Kingdom
}

\{R.Raheem; A.Lasebae; M.Aiash;J.Loo\}@mdx.ac.uk

\begin{abstract}
The dense deployment of Femtocells within the Macrocell's coverage is expected to dominate the future of Long Term Evolution (LTE) networks. While Mobile Femtocells (Mobile-Femtos) could be the solution for vehicular networks when there is a need to improve the vehicular User Equipment (UE) performance by mitigating the impact of penetration loss and path-loss issues. The deployed Femtocells have operated in a co-channel deployment due to the scarcity of spectrums. This issue causes interference between Femtocells and Macrocells as well it causes extra overhead on the LTE networks because of the co-tire interference between adjacent Femtocells. In this paper two interference scenarios are considered, the interference between Mobile-Femto and Macrocell, and the interference between the Mobile Femtos themselves. Therefore, to avoid the generated interference between Femtocells, the controlled transmission powers as well as the coverage planning techniques have been discussed. While in the worst-case scenarios, a frequency reuse scheme has been proposed to avoid the generated interference effectively and dynamically between the Mobile-Femtos as well as their UEs and between the Macrocell UEs.
\end{abstract}

Keywords-LTE; Mobile Femtocell; Co-Channel Interference; Power control; Coverage planning; Fractional Frequency Reuse

\section{INTRODUCTION}

The mobile phone industry has undergone remarkable evolution and has introduced innovative services, leading to a competitive market. Therefore, operators are looking for new technologies that can help to serve this increasing growth in the number of UEs and devices. One of the current methods is to use broadband internet to increase radio coverage and network capacity by deploying Femtocells technologies. However, previous studies have shown that the largest number of phone calls are still made from indoors environment e.g. houses, offices...etc [10] and from vehicular environment e.g. buses and trains [1]. Thus, Femtocell is a technology that can potentially bring large benefits to indoor, outdoor and vehicular wireless communications as well as to network operators and UEs alike. It is also useful to be able to offload traffic from Macrocells down to smaller Base Stations (BSs) such as Femtocells, as this improves the system's capacity and coverage [8]. This might be needed more in the case of celledge vehicular UEs, as those UEs add extra burden on the Macrocell, which in turn wastes the Macrocell resources.
Hence, Femtocells are utilised by service providers as a way of maintaining the quality of service and improving the UE's reception in situations where a high data rate is required e.g. in the case of vehicular UEs. Vehicular UEs experience always high penetration loss and path-loss; therefore, deploying Mobile-Femtos are seen as the future of vehicular environment to improve vehicular UEs' performance [13]. Added to this, the battery life of the mobile device can be prolonged, as it only needs to connect over short distance when connecting to a Mobile-Femto in the same bus, rather than connecting directly to the Macrocell. Due to the Femtocells low power transmission, Femtocells reduce the amount of interference with other electrical devices, while this is not always the case especially when Mobile-Femtos are deployed in the Macrocell and pass closely to each other or closely to a Macrocell. This is because Femtocells might share the same spectrum with the Macrocell or utilise a specific spectrum, and this can be known as a non-orthogonal or an orthogonal mode respectively [2]. Therefore, the deployment of Mobile-Femtos in multi-tier networks requires a high level of interference management scheme because the unplanned dense deployment of Fixed and Mobile Femtos may have a major impact on their characterisation in addition to their identification within the network.

\section{RELATED WORK}

The dense deployment of Femtocells has created a severe interference issue. Therefore, to reduce and mitigate the interference between those small BSs and between the Macrocell, many studies have been carried out based on the power control for Uplink (UL) and Downlink (DL), radio resource scheduling, Femto/Macro local information, UE measurement, cooperative approach, time/frequency allocation, Quality of Service (QoS) requirements, frequency division, frequency reuse and network planning approaches. Several studies have stated that, the position of Femtocells needs to be chosen wisely in order to have the smallest possible number of Femtocells but with the largest possible coverage area [3]. Using this technique reduces the implementation cost and interference. Where other studies have considered the transmission power as a way of controlling the interference like in [4] while in [9], a dynamic power control algorithm is proposed in order to reduce the 
interference level while maximising the indoor coverage. In contrast, in [11], the authors have proposed a decentralised resource allocation scheme for hybrid networks. In this scheme, the available radio resources are divided into time and frequency domains. The Macrocell can select and use all the resources, while the Femtocell has to select only a subset from the available Orthogonal Frequency-Division Multiple Access (OFDMA) frequency resources when it wants to transmit in a random way.

On the other hand, studies like [7] have shown that Femtocells are capable of achieving higher capacity and improving indoor coverage due to the short distance between the transmitter and receiver, where Fractional Frequency Reuse (FFR) scheme has been proposed to improve the spectral efficiency in emerging OFDMA networks. While [1] has proposed a scheme that adjusts its radio frequency parameters taking into account all the network conditions and show that co-channel assignment of the spectrum can lead to higher cell throughputs. However, [6] has proposed a frequency planning mechanism, in which Femtocells choose the frequency sub-bands that will not be used in the sub-region of a Macrocell using FFR in an integrated Macrocell/Femtocell network. In [5], the authors have proposed a novel frequency partitioning method, in which both sub-channels for inner cell region and sub-channels for outer region are allowed to be used in the inner region of cells while sub-channels for outer region are defined differently from cell to cell to reduce Co-Channel Interference (CCI).

\section{INTERFERENCE MANAGEMENT}

The interference management particularly when MobileFemtos are densely deployed in LTE networks is a challenge issue. The densely deployment of those low power vehicular nodes is one of the main features of the future of $4 \mathrm{G}$ networks. It is therefore, vital to the efficiency of the system that the performance of those vehicular nodes does not undermine the activity level of the primary UEs of the system -the Macro UEs- and the secondary UEs of the system -the Femto UEs-. However, the fierce competition for available radio resources among Femtocells is unacceptable because; firstly, it may waste resources, and secondly it does not guarantee the required QoS level. The interference management is even more important when there is a Macro BS that may share all or part of the Bandwidth with the deployed Femtocells. As in this case the already existing Macrocells and UEs may experience interference from the deployed FixedFemtos/Mobile-Femtos as well as the Fixed-Femtos/MobileFemtos may experience interference between each other.

\section{- Coverage Planning Optimisation}

The goal of the coverage planning process is to have good radio conditions everywhere within the outdoor area (especially the cell-edges) or in the vehicular environment so UEs can acquire, initiate and sustain voice calls, as well as overcome the penetration loss and path-loss issues. Good coverage performance is addressed by limiting the maximum path-loss, which can be mitigated by deploying Fixed-Femtos and Mobile-Femtos in the right positions inside the Macrocell and added to this a proper Fixed-Femtos and Mobile-Femtos power calibration is needed. Therefore, it is important to state that the used path-loss model is the Microcell Non Line of Sight (NLOS) path-loss model which can be given by

$$
P L(d B)=34.53+38 \log _{10}(d)
$$

Where $d$ is the distance in meters and it is at least $20 \mathrm{~m}$. On the other hand, the Microcell LOS path-loss model can be given by

$$
\mathrm{PL}(\mathrm{dB})=30.18+26 \log _{10}(\mathrm{~d})
$$

Hence, it became easier to state that the used path-loss on the direct transmission between the evolved Node B (eNB) and the vehicular UEs is the 3GPP Spatial Channel Model (SCM) urban NLOS Microcells model. The same NLOS model has been used for the Fixed-Femto for both; the backhaul link between the eNB and the Fixed-Femto and the access links between the Fixed-Femtos and vehicular UEs. While, in the case of the Mobile-Femto the backhaul link between the Mobile-Femto and the eNB has used the same previous NLOS model while for the access link between Mobile-Femto and the vehicular UE in the same bus has used a 3GPP SCM urban Line of Sight (LOS) Microcells model.

Thus, identifying the used path-loss model between the links of two Base Stations (BSs) or between the links of BSs and vehicular UEs has helped in reducing the interference issue by allowing the operator to know where the Fixed and Mobile Femtocells are needed to be installed. In the case of the Mobile-Femto, identifying the path-loss is an important factor for the network operator because it helps the operator to indicate the Mobile-Femto paths, which are the bus paths. This is due to the fact that, as much as it is important to reduce the path-loss and penetration loss between the vehicular UEs and the serving Femtocell, it is more important to maintain the backhaul path-loss between the eNB and Mobile-Femto, so it can serve those vehicular UEs without losing the connection with the mother BS (eNB) [12].

\section{- Transmission Power control}

On the other hand, controlling the BS transmission power is a very important factor in $4 \mathrm{G}$ systems since it indicates the transmission range of BSs e.g. eNBs and Femtocells. In the Femtocell scenario, as a result of the close distance between the transmitter and the receiver, the channel between the Femtocell and its UEs is considered in a good state which may be less affected by the interfered signals from the eNB. Besides that, the high penetration loss in the case of vehicular UEs due to the vehicle chassis can be counted as an advantage as it prevents the outside signals from passing through to the vehicular UEs. This means that the bus chassis can isolate the vehicular UEs from the outside signals as they can be served 
only by the installed Femtocell (Mobile-Femto) in the same bus, which can help to mitigate the interference.

Thus, indicating the Mobile-Femtos paths, Fixed-Femtos positions and controlling the used transmission power have helped in mitigating the interference, expanding the coverage area and filling the coverage holes. The chosen transmission power in this work has been assumed to be $24 \mathrm{dBm}$ and $46 \mathrm{dBm}$ in the case of Femtocells and eNB respectively. Choosing lower transmission powers has its negative impact on the achieved throughput and number of scheduled UEs even when the interference is mitigated.

\section{PROPOSED SCHEME}

Regarding 4G Femtocells, the mobility of Mobile-Femto positioning in public transportations like buses makes it difficult for the cellular operator to control the interference of these new low power BSs especially when they change their routes (paths). Therefore, an efficient interference mitigation scheme has been proposed for the Mobile-Femto in order to have an interference management and performance improvement. Figure (1) represents three possible interference scenarios. The first interference scenario occurs when a vehicular UE inside bus A interferes with the DL signal of the eNB. This scenario occurs when bus A gets close to the eNB (distance less than 500m) which makes the eNB DL signal strength equal to the DL signal strength of the Mobile-Femto in bus A. On the other hand, the second interference scenario may occur when two nearby Mobile-Femtos interfere with one another e.g. a UE in bus B can be interfered by the DL signal of Mobile-Femto in bus A especially when the used transmission power of the Mobile-Femtos is quite high and vice versa. While the third interference scenario occurs when a Macro UE (primary UE) is close to bus A and interferes by the DL signal of the Femtocell in bus A.

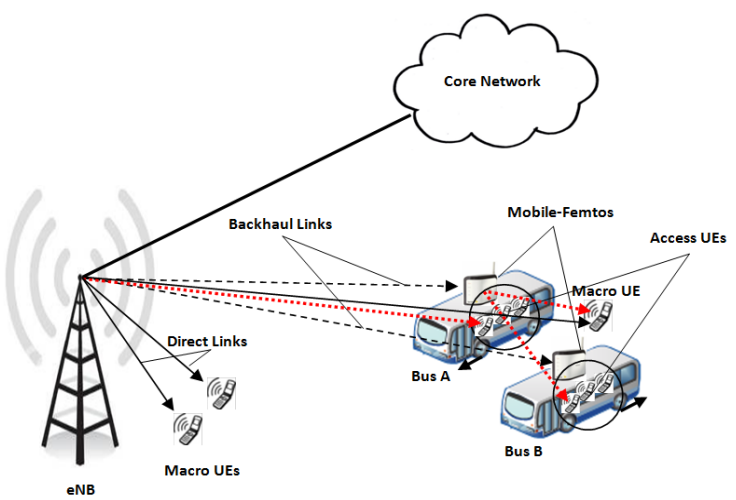

Fig.1 Summarisation of three interference scenarios

To overcome the previous interference issues the FFR scheme has been presented to meet the requirements of the proposed interference management scheme in the Mobile-Femtos deployment. It is to be mentioned that, in the FFR the whole frequency band is divided into several sub-bands, and each sub-band is differently assigned to centre zones and edge zones. The reuse factor of one is used for the centre zone while the edge zone adopts bigger reuse factor. Thus, the Macrocell allocates frequency band using the FFR, the Mobile-Femto chooses sub-bands which have not been used by the Macrocell sub-area and other Mobile-Femtos to avoid the generated interference.

In order to understand the proposed scheme, figure (2) represents the allocated frequency sub-bands to the Macrocells and Femtocells in details. The Macrocell coverage is divided into centre zone and edge zone including the frequency subband $\mathrm{F}_{0}$ for the Macrocell centre zone while the edge zone has three frequency sub-bands denoted by, $F_{1}, F_{2}$ and $F_{3}$. The reuse factor of one is applied in the centre zone, while the edge zone adopts the reuse factor of three. The sub-band $\mathrm{F}_{0}$ is used in the centre zone of Macrocell $_{1}$, Macrocell $_{2}$ and Macrocell $l_{3}$, and sub-band $F_{1}, F_{2}$ and $F_{3}$ is applied in the edges of Macrocell $_{1}$, Macrocell 2 and Macrocell ${ }_{3}$ respectively. Under this circumstance, the Mobile-Femto chooses sub-bands which have not been used by the Macrocell sub-area and other Mobile-Femtos as mentioned earlier.

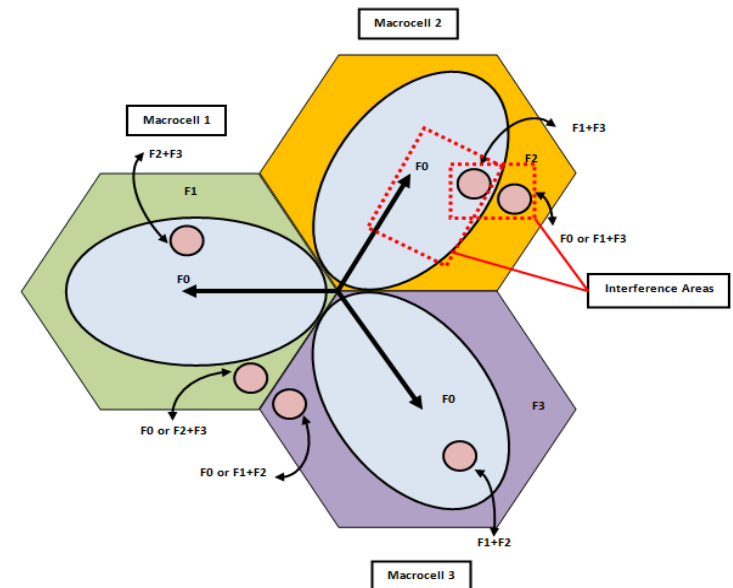

Fig. 2 The proposed interference management scheme based on the FFR

The proposed scheme receives as an input the Macrocell environment dimensioning, the number of Mobile-Femtos and their positions as well as other characteristics of the network such as the Macrocell and Mobile-Femtos transmission powers. Then, it calculates the received power from the serving cells as well as from the interfering cells. Based on the pervious values and by taking into account the white Gaussian noise, the scheme is able to make estimation for the Signal-toInterference-plus-Noise Ratio (SINR) and throughput at any given position of the examined LTE network. Thus, the proposed scheme follows the approach presented below:

\section{Calculate the inner cell radius}

Based on the Macrocells' characteristics and the used transmission power, the inner cell radius can be calculated which will help later to indicate the centre zone and cell-edge zone as figure (3) shows. 


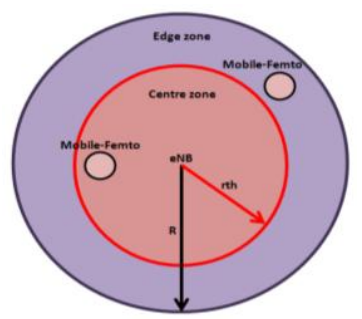

Fig.3 Inner Cell radius

\section{Find the optimum frequency band division}

The aim of this step is to calculate the UEs throughput as well as the UEs SINR for every possible combination of the spectrum division. The radio spectrum is divided into frequency sub-bands reserved for a single use or a range of compatible uses. Within each band, individual transmitters often use separate frequencies or channels, so they do not interfere with each other. Hence, the available spectrum will be allocated to UEs according to the combination that maximises their throughput. Thus, there are two disjoint sets of subcarriers: the subcarriers in the centre zone and the subcarriers in the cell-edge zone. Every time the frequency band that is allocated to the cell-edge zone is equally divided between $F_{1}, F_{2}$ and $F_{3}$ as mentioned earlier. Initially, let it be assumed that the set of the centre zone is an empty set and all the subcarriers are contained in the set of cell-edge zone, that means all the subcarriers that are allocated to the cell-edge zone, and each one of $F_{1}, F_{2}$ and $F_{3}$ equals to Total $1_{\text {subcarriers }} / 3$. Each time one subcarrier is removed from the set of cell-edge zone and added to the set of centre zone. Finally, the set of the centre zone will consist of Total ${ }_{\text {subcarriers }}$ and the set of the celledge zone will be an empty set.

\section{Allocate frequency band to the Mobile-Femtos}

In this step, the frequency is allocated to the Mobile-Femtos with the process presented in figure (2). However, the proposed scheme has been summarised in Algorithm 1.

\section{Algorithm1:Frequency Reuse for Macro/MFemto Interference Avoidance}

/*frequency band division allocate frequency band for macrocells; if $r>=$ distance_mfemto $/ *$ Mobile-Femto belongs to Macro centre zone

$$
\text { allocate frequency band for mfemto; }
$$

While in order to understand the impact of implementing the proposed interference avoidance scheme on the UEs performance, the following section presents the required mathematical equations that will help later to evaluate the system performance.

\section{SYSTEM PERFORMANCE ANALYSIS}

Hence, the received SINR for an outdoor direct Macro UE (m) on subcarrier $\mathrm{n}$ can be expressed as

$$
\operatorname{SINR}_{\mathrm{m}, \mathrm{n}}=\frac{\mathrm{P}_{\mathrm{x}}^{\mathrm{eNB}} \mathrm{G}_{\mathrm{m}, \mathrm{eNB}, \mathrm{n}} \beta \mathrm{x}^{-\alpha} \varepsilon}{\sum_{\mathrm{l=1,l \neq 1}}^{\mathrm{eNB}} \mathrm{P}_{\mathrm{x}}^{\mathrm{eNB}} \mathrm{G}_{\mathrm{m}, \mathrm{eNB}, \mathrm{n}}+\sum_{\mathrm{j}=1}^{\mathrm{MFemto}} \mathrm{P}_{\mathrm{x}}^{\mathrm{MFemto}} \mathrm{G}_{\mathrm{m}, \mathrm{MFemto}, \mathrm{n}}+\mathrm{P}_{\text {noise }} \Delta \mathrm{f}}
$$

Where, $\mathrm{P}_{\mathrm{x}}^{\mathrm{eNB}}$ and $\mathrm{P}_{\mathrm{x}}^{\mathrm{eNB}}$, are the transmission power of the serving Macrocell (eNB) and the neighbouring Macrocell (eNB') on subcarrier $n$ respectively. $G_{m, e N B, n}$ is the channel gain between the Macro UE $m$ and serving Macrocell (eNB) on subcarrier $n$. Channel gain from neighbouring Macrocells is denoted by $G_{m, e N B^{\prime}, n}$. Similarly, $P_{x}^{M F e m t o ~ i s ~ t h e ~ t r a n s m i s s i o n ~}$ power of neighbouring Mobile-Femto (MFemto) on subcarrier n. $G_{m, M F e m t o, n}$ is the channel gain between the Macro UE $\mathrm{m}$ and neighbouring Mobile-Femto (MFemto) on subcarrier n. Whilst, $\beta x$ has been modelled to express the pathloss model as $\beta$ here is the path-loss constant, $\mathrm{x}$ is the distance between the Macro UE and the serving eNB and $\alpha$ is the pathloss exponent. $\varepsilon$ is the Vehicular Penetration Loss (VPL), $\mathrm{P}_{\text {noise }}$ is the white noise power, and $\Delta \mathrm{f}$ is subcarrier spacing.

In contrast, the Macrocell or any adjacent Mobile-Femto can cause interference to a Mobile-Femto's UE. Thus, the received SINR of a Mobile-Femto UE (mf) on subcarrier $\mathrm{n}$ can be formulated as

$$
\operatorname{SINR}_{\mathrm{mf}, \mathrm{n}}=\frac{\mathrm{P}_{\mathrm{x}}^{\mathrm{MFemto}} \mathrm{C}_{\text {loss }}}{\sum_{\mathrm{i}=1}^{\mathrm{eNB}} \mathrm{P}_{\mathrm{x}}^{\mathrm{eNB}} \mathrm{G}_{\mathrm{mf}, \mathrm{eNB}, \mathrm{n}}+\sum_{\mathrm{v}=1, \mathrm{v} \neq \mathrm{j}}^{\mathrm{MFemto}} \mathrm{P}_{\mathrm{x}}^{\mathrm{MFemto}} \mathrm{G}_{\mathrm{mf}, \mathrm{MFemto}, \mathrm{n}}+\mathrm{P}_{\text {noise }} \Delta \mathrm{f}}
$$

There is a system constant loss but no channel gain over the Mobile-Femto access link between the UE and the serving Mobile-Femto as the distance between the two is very short (less than $5 \mathrm{~m})$. The only existing channel gain $\left(\mathrm{G}_{\mathrm{mf}, \mathrm{MFemto}, \mathrm{n}}\right)$ is the channel gain between the Mobile-Femto UE with other adjacent Mobile-Femtos that may cause interference to the considered UE. Hence, the Macro-UE capacity on a specific subcarrier $n$ can be estimated via the SINR from the following equation

$$
\mathrm{C}_{\mathrm{m}, \mathrm{n}}=\mathrm{BW} \cdot \log _{2}\left(1+\alpha \mathrm{SINR}_{\mathrm{m}, \mathrm{n}}\right)
$$

Where $\mathrm{BW}$ is the available bandwidth for subcarrier $n$ divided by the number of UEs that share the specific subcarrier and $\alpha$ in this equation is a constant for target Bit Error Rate (BER) defined by $\alpha=-1.5 / \ln (5 \mathrm{BER})$. Here, the BER has been set to $10^{-6}$. So the overall throughput of the serving Macrocell $\mathrm{M}$ can be expressed as 


$$
\text { Throughput }_{\mathrm{M}}=\sum_{\mathrm{m}} \sum_{\mathrm{n}} \beta_{\mathrm{m}, \mathrm{n}} \mathrm{C}_{\mathrm{m}, \mathrm{n}}
$$

Where, $\beta_{\mathrm{m}, \mathrm{n}}$ here notifies the subcarrier assignment for Macro UEs. When $\beta_{\mathrm{m}, \mathrm{n}}=1$, the subcarrier $\mathrm{n}$ is assigned to Macro UE $\mathrm{m}$ and otherwise $\beta_{\mathrm{m}, \mathrm{n}}=0$. From the characteristics of the OFDMA system, each subcarrier is allocated to only one Macro UE in a Macrocell in every time slot. This implies that $\sum_{m=1}^{N_{m}} \beta_{m, n}=1$ for $\forall \mathrm{k}$, where $\mathrm{N}_{\mathrm{m}}$ is the number of Macro UEs in a Macrocell and $\mathrm{k}$ is the available recourse blocks. While similar expression for Mobile-Femto UEs related to the practical capacity and the overall throughput is possible except $\sum_{m f=1}^{N_{m f}} \beta_{m f, n}=1$ for $\mathrm{k} \in \mathrm{F}_{\text {Mobile-Femto. }} \mathrm{N}_{\mathrm{mf}}$ is the number of Mobile-Femto UEs in a Macrocell and $F_{\text {Mobile-Femto is the }}$ available sub-bands allocated to Mobile-Femtos in Macrocells. This implies that the proposed scheme reuses the full frequency band between the Macrocells and Mobile-Femtos. Thus, the Mobile-Femto UE capacity on a specific subcarrier $\mathrm{n}$ can be estimated via the SINR as the following equation shows

$$
\mathrm{C}_{\mathrm{mf,n}}=\mathrm{BW} \cdot \log _{2}\left(1+\alpha \mathrm{SINR} \mathrm{mf,n}_{\mathrm{n}}\right)
$$

Where the overall throughput of the serving Mobile-Femto (MFemto) can be expressed as

$$
\text { Throughput }_{\mathrm{MFemto}}=\sum_{\mathrm{mf}} \sum_{\mathrm{n}} \beta_{\mathrm{mf}, \mathrm{n}} \mathrm{C}_{\mathrm{mf}, \mathrm{n}}
$$

After discussing the SINR, throughput and capacity, here it is important to state that the outage probability plays an essential role in the proposed scheme as it affects the network performance by affecting the data rate and throughput of UEs. If the outage probability is small, the throughput increases, and then when the throughput increases the performance improves and the interference decreases.

However, the outage probability affects the performance of cell-edge UEs more than the Macro UEs due to their high path-loss. To find out the outage probability it is required first to identify the SINR threshold value in the range of $0 \mathrm{~dB}$ to $30 \mathrm{~dB}$. Thus, the outage probability $\left(\mathrm{P}_{\text {out }}\right)$ is determined when the SINR level of a subcarrier does not exceed the designated threshold and it can be given by

$$
\mathrm{P}_{\mathrm{out}}=\frac{\sum_{\mathrm{u}} \sum_{\mathrm{n}} \delta_{\mathrm{u}, \mathrm{n}} \cdot \operatorname{SINR}_{\mathrm{u}, \mathrm{n}}}{\sum_{\mathrm{u}} \sum_{\mathrm{n}} \beta_{\mathrm{u}, \mathrm{n}} \cdot \operatorname{SINR}_{\mathrm{u}, \mathrm{n}}}
$$

Where, $\delta_{u, n}$ indicates the failed subcarrier assignment for UE $\mathrm{u}$ on subcarrier $\mathrm{n}$. If $\delta_{u, n}=1$, the SINR of that subcarrier is under the SINR threshold $\left(\operatorname{SINR}_{\mathrm{u}, \mathrm{n}}<\mathrm{SINR}_{\text {threshold }}\right)$. Thus, the ratio between the number of subcarriers under the SINR threshold and the number of the total subcarriers is the outage probability.

The subsequent results will show that the proposed scheme is greatly capable of preventing the interference among the Macro UEs as well as the interference among the MobileFemto UEs. The Mobile-Femto UEs effect on Macro UEs and vice versa is less in the proposed scheme in comparison with the previous schemes. For example, the FFR-3 (frequency factor reuse 3) and NoFFR (frequency factor reuse 1) techniques assign random subcarriers to the Femto UEs, regardless of the subcarriers that have been used by the Macro UE. Therefore, the Macro UEs and the Femto UEs may use subcarriers very nearer to one another that cause interference.

\begin{tabular}{|c|c|c|c|c|}
\hline \multirow[b]{2}{*}{ Schemes } & \multicolumn{2}{|c|}{ Macro-user } & \multicolumn{2}{|c|}{ Femto-user } \\
\hline & Frequency & Amount & Frequency & Amount \\
\hline Proposed & FFR & 2 & $\begin{array}{c}\text { Divide centre } \\
\text { and edges } \\
\text { zones }\end{array}$ & 3 \\
\hline $\begin{array}{c}\text { FFR-3 } \\
\text { (reuse 3) }\end{array}$ & FFR & \multirow[t]{2}{*}{1} & Random & \multirow[t]{2}{*}{1} \\
\hline $\begin{array}{r}\text { NoFFR-3 } \\
\text { (reuse 1) }\end{array}$ & Random & & Random & \\
\hline $\begin{array}{l}\text { Note: Amo } \\
\text { Macro and }\end{array}$ & $\begin{array}{l}\text { olumn in } \\
\text { le-Femto }\end{array}$ & $\begin{array}{l}\text { value of } \\
\text { espectively }\end{array}$ & $\beta_{m n}$ and $\sum^{N_{r}}$ & $\beta_{m f, n}$ for \\
\hline
\end{tabular}
The above has been summarised in Table (1).

Table1: Comparison between the proposed and previous interference management schemes

\section{RESULTS AND DISCUSSION}

Since vehicular UEs are always suffering from high pathloss, high VPL and shadow fading, implementing Femtocells (Fixed and Mobile) can be a reasonable solution to overcome the previous issues. However and due to vehicular UEs high mobility, the coverage distribution and signal transmission power of Fixed-Femtos and Mobile-Femtos play important roles in improving the UEs performance as well as reducing the interference issue as much as possible. Therefore, the following two scenarios represent the coverage distribution based on the used path-loss model as well as the assumed transmission power of Fixed-Femtos and Mobile-Femtos.

The first figure represents the Fixed-Femtos deployment to serve vehicular UEs in LTE Networks. In this figure, the use of high transmission power, which is $24 \mathrm{dBm}$ to reach the vehicular UEs and overcome the high VPL, is quite obvious. However, this has created overlapping areas (interference areas).

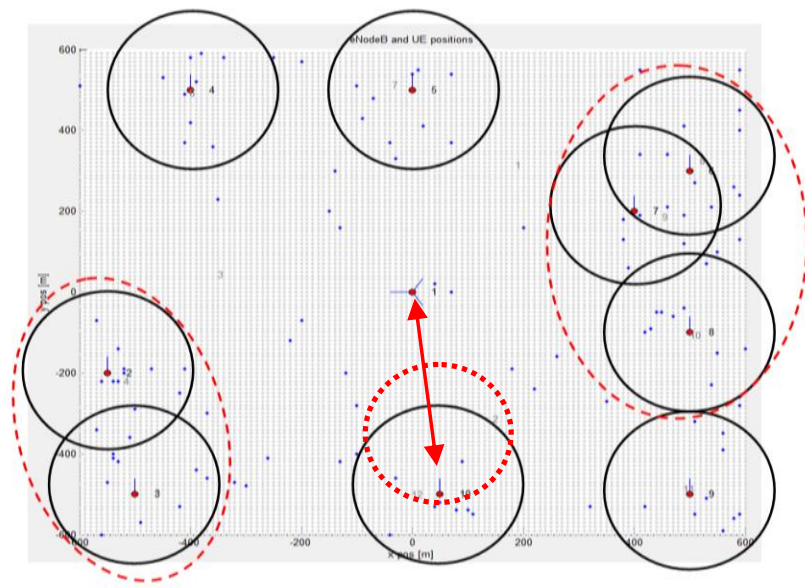

Fig.4 Fixed-Femtos coverage distribution with 25dB VPL 
While Figure (5) represents the Fixed-Femtos distribution in the Region of Interest (ROI) in term of path-loss with and without shadow fading. It is noticeable that there are many overlapped areas due to the high transmission power of those Fixed-Femtos to reach the far vehicular UEs in the coverage area of eNB as well as to overcome the high VPL.

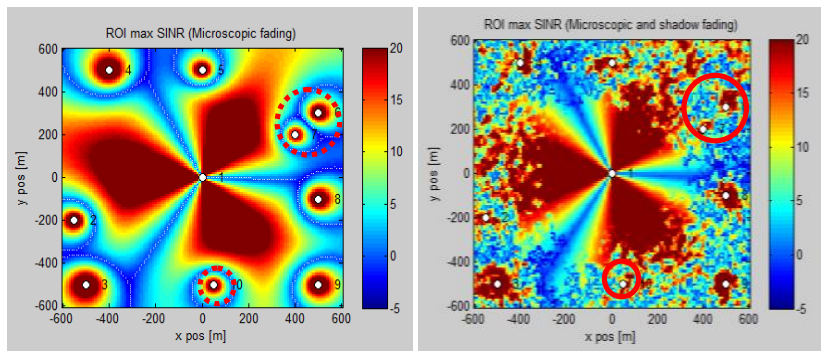

Fig.5 Fixed-Femtos Sector SINR vs. path-loss without and with shadow fading

The overlapped areas do not only have a negative impact on the SINR but also on the spectral efficiency as figure (6) shows. It is obvious that the signal strength of the interfered areas fades faster than other Fixed-Femtos because of the interference effect on the signal strength as well as the achieved spectral efficiency.

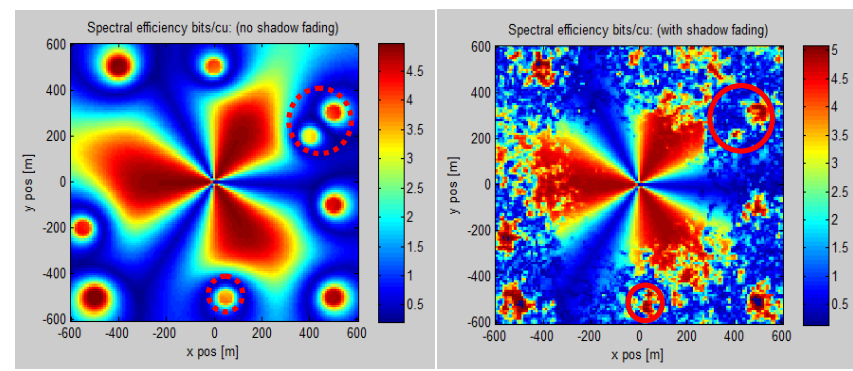

Fig.6 Fixed-Femtos Sector Spectral efficiency vs. path-loss without and with shadow fading

In order to mitigate the interference issue between the Macro and Fixed-Femto UEs, the transmission power of these FixedFemtos can be reduced to avoid the overlapped areas. Reducing the transmission power can limit the coverage area of the Fixed-Femto, which may increase the cost since more Fixed-Femtos are required to be deployed to cover all the high path-loss areas. Moreover, reducing the transmission power can increase the number of unnecessary Handovers (HOs) especially in the case of vehicular UEs.

In contrast, the second scenario represents the case when there is Mobile-Femto installed in a bus while the UEs are inside the bus. The aim of this scenario is to improve the signal connection of those vehicular UEs with the serving network. Thus, installing the Mobile-Femtos efficiently in public transportations like buses has helped in mitigating the VPL, path-loss and cost to serve vehicular UEs. A LOS Microcell path-loss model has been implemented between the Mobile-Femtos and vehicular UEs due to the short distance between the two. In addition, the used transmission power in the Mobile-Femto is $24 \mathrm{dBm}$ while the path of the MobileFemto has been specified, and two Mobile-Femtos have been assumed to be deployed in every $1 \mathrm{Km}^{2}$. Using this technique has its advantages in mitigating the interference and the pathloss issues. This has given the Mobile-Femto the ability to cover wider areas that suffer from high VPL and path-loss.

Hence, figure (7) shows the movement of the Mobile-Femto, which is a uniform movement in the areas that suffer from high path-loss as the arrows show.

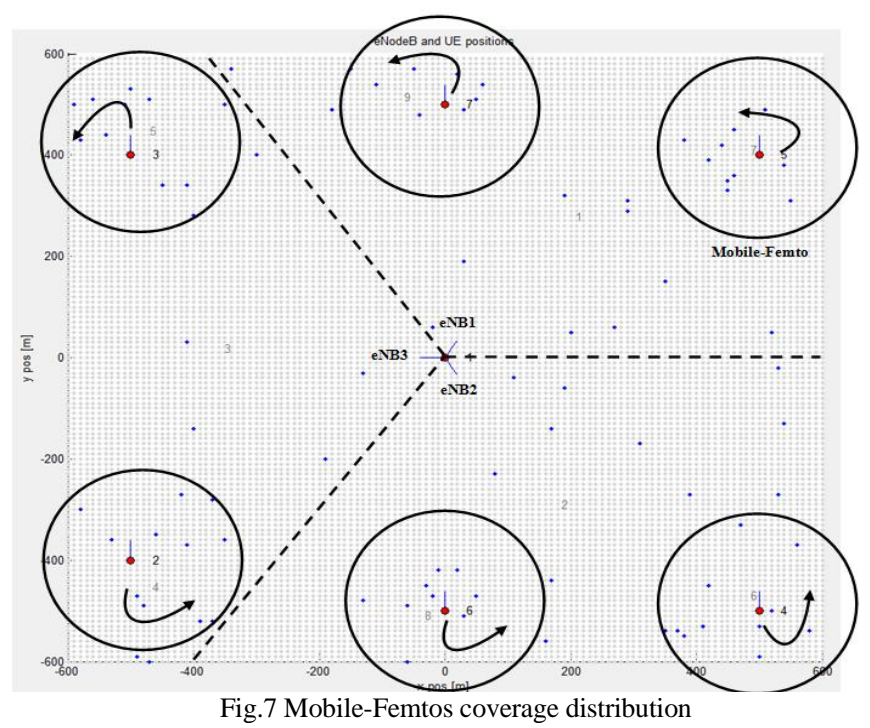

The max SINR of the ROI has been improved after deploying the Mobile-Femtos to serve vehicular UEs. This is because Mobile-Femtos are able to overcome the high VPL, path-loss and interference issues that vehicular UEs are suffering from compared to the Fixed-Femto deployment as figure (8) shows. It has become quite obvious that Mobile-Femtos have achieved better coverage area as the paths of these MobileFemtos were specified. The figure shows that each MobileFemto has strong signal strength at the centre then the signal fades gradually.

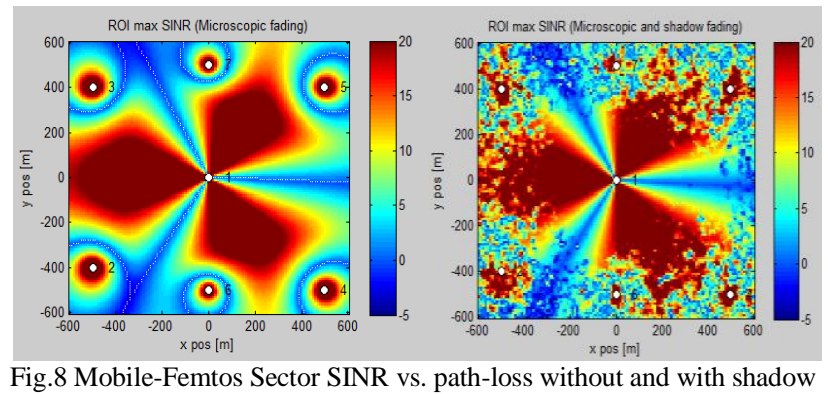
fading

The SINR improvement is accompanied with an improvement in the achieved spectral efficiency as figure (9) shows. The high spectral efficiency reflects that the Mobile-Femto coverage is less susceptible to interference compared to the Fixed-Femto. This is due to the organised distribution and the specified paths in the case of the Mobile-Femto, which makes it less exposed to the interference issue. 


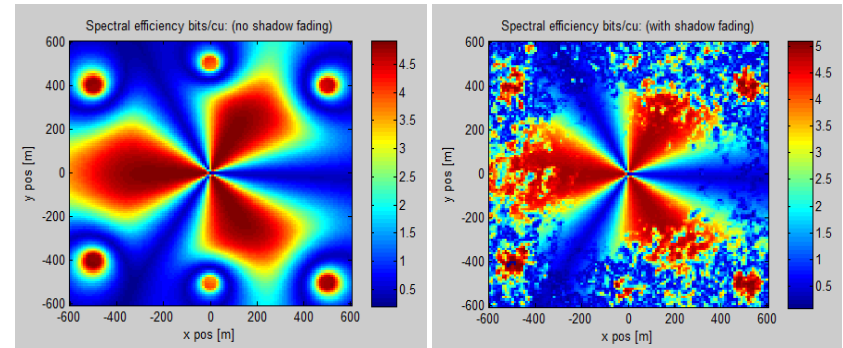

Fig.9 MobileFemtos Sector Spectral efficiency vs. path-loss without $\&$ with shadow fading

On the other hand, worst-case scenarios are always expected therefore here it comes the results of the proposed interference management scheme compared to previous schemes. Figure (10) represents the throughput of Macro UEs as the number of Mobile-Femtos varies. In the proposed scheme, the MobileFemto UEs can allocate the subcarriers, which are not used by the Macro UEs at each location. Thus, the interference between the Macro UEs and Mobile-Femtos is greatly avoided. The Mobile-Femtos effect to Macro UEs who are located in the same zone is less in the proposed scheme compared to the previous schemes. This is because in FFR-3 and NoFFR-3 schemes, the subcarriers are randomly assigned to the MobileFemto UEs regardless of the subcarriers used by the Macro UEs.

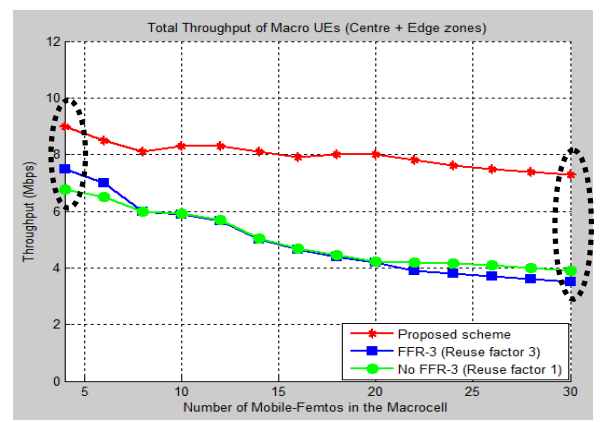

Fig.10 Total throughput of Macro UEs located in centre \& edge zones vs. No. of Mobile-Femto

While figure (11) illustrates the throughput of Macro and Mobile-Femto UEs located at the edge zone only. In the OFDMA cellular network, the performance of the edge zone is always poor due to the Inter-Cell Interference (ICI) that increases with the increased path-loss.

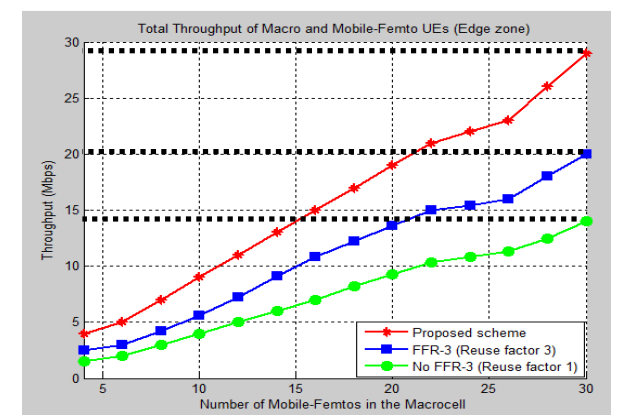

Fig.11 Total cell-edge UEs throughput vs. number of Mobile-Femto
However, in the proposed scheme, allocating more subcarriers to cell-edge Mobile-Femtos can greatly improve the throughput of cell-edge vehicular UEs. While, the compared schemes assign the subcarriers irrespective of the UEs location, centre zone or edge zones and this is why those schemes achieved less throughput compared to the proposed scheme.

Whilst figure (12) represents the total throughput of centre and cell-edge zones after deploying the Mobile-Femto in the Macrocell. The achieved throughput increases with the increased number of deployed Mobile-Femtos in the Macrocell. However, deploying many Mobile-Femtos to improve the vehicular UEs performance in LTE network can increase the interference issue between Macro and MobileFemto UEs. Therefore, implementing the proposed scheme has shown better throughput compared to FFR-3 and No-FFR schemes.

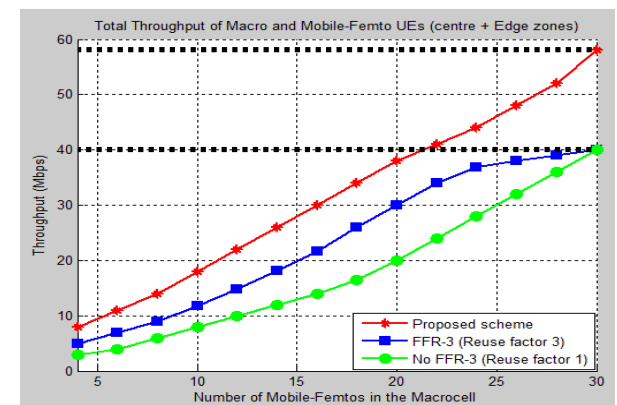

Fig.12Total throughput of Macrocell \& Mobile-Femto UEs vs. No. of MobileFemtos

Now, after discussing the impact of the proposed scheme on the achieved throughput of Macro and Mobile-Femto UEs, it is necessary to consider the impact of this scheme on the achieved signal outage probability with the increased number of Mobile-Femtos in the Macrocell. It is to be mentioned that with the increased number of deployed small BSs in the Macrocell, the generated interference issue increases, thus the signal outage increases as well. However, this does not mean that less number of Mobile-Femtos should be deployed in the Macrocell to mitigate the generated interference and signal outage. The signal outage can be reduced with the proposed scheme as figure (13) shows. The figure depicts the outage probability of UEs according to the SINR threshold, when 30 Mobile-Femtos are deployed in the Macrocell. In a given SINR threshold, the proposed scheme indicates lower outage probability than the FFR-3 and NoFFR-3 schemes. Added to this, it decreases the outage probability more at low SINR threshold while it reaches its max at 0.96 with high SINR threshold. This implies that the proposed scheme effectively supports more UEs, even though the interference is severe.

While in the case of the NoFFR-3, UEs have achieved the highest outage probability, which has started with 0.4 under low SINR threshold and continuously increased with the increased SINR threshold. However, at $15 \mathrm{~dB}$ SINR the outage probability of the proposed scheme has been reduced by 0.07 
compared to the FFR-3 scheme while reduced by 0.14 compared to the NoFFR-3 scheme.

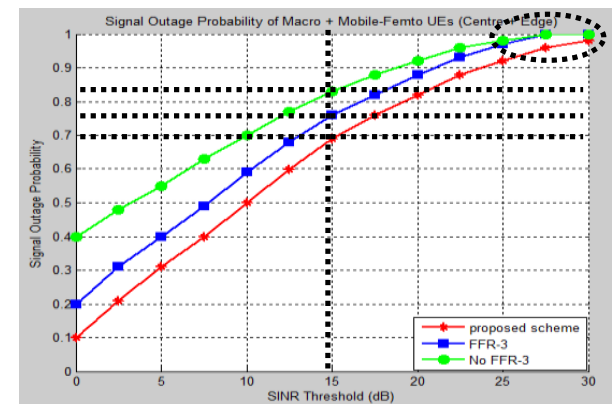

Fig.13 Signal Outage Probability of Macro and Mobile-Femto UEs when 30 Mobile-Femtos are deployed in the Macrocell

Thus, it is obvious that using the proposed frequency reuse scheme in the presented Femtocell technology has improved the UEs throughput in the cell centre and edge zones for both Macro and Mobile-Femto UEs. It also has reduced the outage probability of vehicular UEs in compared to other frequency reuse schemes.

\section{CONCLUSION}

This paper has introduced the interference issues that small BSs can cause in LTE networks. This interference is worst in the case of cell-edge vehicular UEs due to the high VPL, pathloss and shadowing. As a result, controlling the transmission power as well as optimising the BS distribution in the network can cause positive or negative impacts on the generated interference in LTE networks. However, there are always worst-case scenarios that require special mechanisms to mitigate the generated interference between Macro and Mobile-Femto UEs. Accordingly, the proposed interference mitigation scheme based on the frequency reuse scheme has achieved an obvious throughput gain in term of Macro and Mobile-Femto UEs in the cell centre and cell-edge zones compared to previous schemes e.g. FFR-3 and No FFR-3. It also has reduced the outage probability of vehicular UEs and improved the quality of their connections with the serving BSs.

\section{ACKNOWLEDGMENT}

The authors would like to thank all those who contributed to the completion and success of this work. Also, the Science and Technology department at Middlesex University played a significant role in supporting and backing this work at all stages of the study.

\section{REFERENCES}

[1] 5GNow, $5^{\text {th }}$ Generation Non-Orthogonal Waveforms for Asynchronous Signalling, accessed on: 15/05/2015, available at: http://www.5gnow.eu.

[2] S. Al-Rubaye, "Radio Network management in Cognitive LTE/Femtocell Systems", Ph.D thesis, Brunel University, Sep 2012.

[3] M. Bennis \& L. Giupponi, "Interference management in self-organized femtocell networks: The BeFEMTO”, Wireless VITAE Conference, 2011.

[4] H. Claussen, "Performance of macro and co-channel femtocells in a hierarchical cell structure", PIMRC IEEE 18 ${ }^{\text {th }}$ international Symposium, 2007.

[5] S. Han \& J. Park, "A new frequency partitioning and allocation of subcarriers for FFR in mobile communication systems", IEICE, 2008.

[6] P. Lee, T. Lee, J. Jeong, and J. Shin, "Interference management in LTE femtocell systems using fractional frequency reuse", ICACT, Korea, 2010.

[7] A. Mahmud and k. A. Hamdi, "On the Co-channel Femtocells Exclusion Region in FFR Macrocells", WCNC, Istanbul, 2014.

[8] N. Saquib, E. Hossain, L. Le and D. Kim, "Interference management in OFDMA femtocell networks: Issues and approaches", Wireless Com, 2012.

[9] D. Shin and S. Choi, "Dynamic power control for balanced data traffic with coverage in femtocell networks", in IWCMC $8^{\text {th }}$ international, 2013.

[10]W. Wang and Q. Zhang, "Local cooperation architecture for self-healing femtocell networks", Wireless Com, IEEE, 2014.

[11]X. Chu, Y. Wu and L. Benmesbah, "Resource Allocation in Hybrid Macro/Femto Networks", in IEEE WCNCW, 2010.

[12]R. Raheem, A. Lasebae \& J. Loo, "Mobility Management in LTE/Mobile Femtocell Networks: Outage probability \& Drop/Block Calls probability", ERPBSS-UAE 2015.

[13]R. Raheem, A. Lasebae, M. Aiash and J. Loo, "Performance Evaluation of Mobile Users Served by Fixed and Mobile Femtocells in LTE Networks", in proc. of the International Journal of Network Technology, 2016. 